\title{
Critical Current Anisotropy in Conventional and Artificial Pinning Center Round Wire Nb-Ti Superconductors
}

\author{
Cristina Bormio Nunes,* Robert W. Heussner and David C. Larbalestier \\ Applied Superconductivity Center, University of Wisconsin-Madison, WI 53706 \\ *on leave from Faenquil/Demar, 12600-000 Lorena SP, BRAZIL
}

\begin{abstract}
Critical current density anisotropy was detected in conventional and artificial pinning center (APC) $\mathrm{Nb}-\mathrm{Ti}$ round wires by means of a relevant increase of the magnetization $M$ as the wire aspect ratio $A_{w}$ (length $L /$ filament diameter $d_{f}$ ) was increased. However, contrary to what is observed in APC wires, the critical current density $J_{||}$calculated from the SQUID measured saturated-magnetization in conventional wires using an anisotropic Bean Model (BM), is significantly different from the transport critical current density $\mathrm{J}_{\mathrm{ct}}$. We suggest that the mismatch between SQUID measured $J_{\mid}$and $J_{c t}$ is due a combination of the discontinuity of the ribbons along the wire length in conventional $\mathrm{Nb}-\mathrm{Ti}$ wires and also to the low electric fields generated in SQUID measurements. The low electric fields allows the magnetization-induced currents to redistribute inside the wire due to the local inhomogeneities associated with the presence of $\alpha$-Ti ribbons. In this case anisotropic BM cannot be used to extract $J_{c}$ from $M$ because it will be function of the intrinsic lengths of these ribbons.
\end{abstract}

\section{INTRODUCTION}

It is well known that a strong flux pinning microstructure can be created in $\mathrm{Nb}-\mathrm{Ti}$ superconductors by introducing a second phase into the matrix, either by sequences of heat treatment followed by cold deformation [1] or artificially [2],[3],[4] by sequences of bundling, extrusion and cold drawing. These two processes produce so-called conventional and artificial pinning center (APC) superconducting wires respectively. Such wires develop a nanostructure containing long, thin ribbons aligned parallel to the wire axis.

Previous magnetization measurements of $\mathrm{Nb}-\mathrm{Ti} \mathrm{APC}$ wires with $\mathrm{Nb}$ pins have shown that the hysteretic magnetization $(\Delta \mathrm{M})$ increases as the wire length (L) is increased [5], for a fixed superconductor filament diameter $d_{f}$. This effect is a consequence of the anisotropy of the flux pinning nanostructure of elongated ribbons. There are two features that contribute to the critical current density anisotropy. One is that the current which flows axially along the wire and parallel to the ribbons $\left(J_{\|}\right)$is intrinsically different from the current that crosses the ribbons to complete the magnetization current loop $\left(\mathrm{J}_{\perp}\right)$. The second is that the pinning force $\left(\mathrm{F}_{\mathrm{p}}\right)$ that opposes the Lorentz force on the vortex lattice is much stronger for $J_{\|}$than for $J_{\perp}$ because the ribbons are effective barriers against vortex movement only for $\mathrm{J}_{\|}$. In [5] we calculated the critical current density anisotropy $J_{||} / J_{\perp}$ vs. $H$ from $\Delta M(L)$ vs.

Manuscript received August 27, 1996

This work was supported by the U.S. Department of Energy-Division of High Energy Physics. C.B. Nunes is supported by a fellowship from CNPq/Brazil.
$\mathrm{H}$ measurements using an anisotropic Bean Model (BM) for cylindrical samples [6]. For the particular $\mathrm{Nb}$-pin $\mathrm{APC} \mathrm{Nb}-$ Ti wires analyzed in [5], the $J_{c}$ anisotropy $J_{/ /} / J_{\perp}$ reached values as high as 175. Another important conclusion from the $\Delta \mathrm{M}(\mathrm{L})$ vs. $\mathrm{H}$ measurements and the subsequent anisotropic $\mathrm{BM}$ analysis was that the technologically important critical current density $J_{c}=J_{\|}$(the transport critical current $J_{c t}$ ) can be calculated from magnetization data using the simple isotropic $B M$ [7] only for rather long wires, such that $L / d_{f}>J_{\mid} / J_{\perp}$. This condition corresponds to the saturation of $\mathrm{M}(\mathrm{L})=\mathrm{M}_{\mathrm{s}}$, meaning that for lengths $\mathrm{L}>\mathrm{L}_{s}$, $\mathrm{M}$ no longer increases as the length of the wire is increased. Here we call $\mathrm{L}_{\mathrm{s}}$ the saturation length and $\mathrm{M}_{\mathrm{s}}$ the saturation magnetization.

The objective of the present work was to evaluate the anisotropy of the critical current density also in conventional $\mathrm{Nb}-\mathrm{Ti}$ optimized wires from sample-length-dependent $\Delta \mathrm{M}(\mathrm{H})$ measurements. We sought any differences in $J_{\mid} / \mathrm{J}_{\perp}$ vs. $\mathrm{H}$ and $\mathrm{J}_{\perp}$ vs. $\mathrm{H}$ between conventional and $\mathrm{APC} \mathrm{Nb-Ti} \mathrm{wires}$ because, in spite of the fact that the optimized nanostructures have similar geometry [1], [8], the pin materials are quite different, $\mathrm{Nb}$ in the APC and $\alpha-\mathrm{Ti}$ in conventional wires. Determination of the current density that crosses the pins $J_{\perp}$ should bring valuable information about the effect of pin material on the flux pinning properties.

The $\Delta M(L)$ vs. $H$ measurements were made on optimized conventional $\mathrm{Nb}-\mathrm{Ti}$ wires using a SQUID magnetometer. They also showed a dependence of the magnetization on the wire length. However, we observed that the axial critical current density $\mathbf{J}_{\|}$calculated using the isotropic BM from $M_{s}$ was significantly different from the transport critical current density $J_{c t}$. On the other hand, for APC wires there was no difference between $\mathrm{J}_{\mathrm{ct}}$ and the current density calculated from $\mathrm{M}_{s}$ measured in a SQUID magnetometer.

This surprising difference in behavior caused us to think harder about the distinctions between APC and conventional wires, about electric field effects and about the potential current paths in APC wires with ribbon-shaped pins which run the full length of the wire ( $\mathrm{mm}$ to $\mathrm{m}$ ), as compared to conventional wires whose $\alpha$-Ti ribbons are only of order $10 \mu \mathrm{m}$ in length. The low electric fields generated in the samples during SQUID measurement makes it possible for the magnetization induced-currents to redistribute between individual ribbons where one ribbon ends and another starts [1]. The interpretation of the magnetization data in this case should depend on the dimensions (particularly the length) of the ribbons, meaning that they have intrinsic longitudinal $\mathrm{L}_{\mathrm{i}}$ and 
transverse $d_{i}$ lengths. The magnetization may no longer be calculated, even using the anisotropic BM.

In section II and III we present a short description of the experiment and how to calculate or measure $J_{\|}$and $J_{\perp}$ using the principles of the anisotropic BM, respectively. The results obtained from SQUID and transport critical current $\left(\mathrm{J}_{\mathrm{ct}}\right)$ measurements showing the dependence of magnetization on wire length for conventional wire and also the comparison between $J_{c t}$ and $J_{||}$(SQUID) are discussed in section IV. In section $\mathrm{V}$ the critical current density anisotropy ratios for conventional and APC $\mathrm{Nb}-\mathrm{Ti}$ wires are compared using transport and magnetization data. In section VI the results are summarized.

\section{EXPERIMENTAL DESIGN}

\section{A. Wire Description}

The samples were selected from three $\mathrm{Nb}-\mathrm{Ti}$ wires. One is an $\mathrm{APC}$ wire with $\mathrm{Nb}$ pins (sample $\mathrm{A}$ ) while the other two are conventional wires (samples B and C). Samples A and B are monofilaments and sample $C$ is a 61 multifilament strand. Sample B was studied for two different superconductor filament diameters, named B1 and B2. The characteristics of each wire are shown in Table I (superconductor filament diameter $d_{f}$, the measured pinning center thickness $t_{p}$ and also the wire aspect ratio $A_{w}$ ). The actual pin thickness $t_{p}$ was measured from transmission electron microscope (TEM) images of A, B1 and B2. For all wires, the matrix is a high homogeneity $\mathrm{Nb} 47 \mathrm{wt} \%$ Ti alloy.

Sample A, the APC wire, has a pin volume fraction of $24.4 \%$. It was fabricated using the rod-based process describe previously [4], [7].

Samples B and C, the conventional wires, have $\alpha$-Ti ribbons as pins. Sample B has $\sim 19$ vol. $\%$ of $\alpha$-Ti precipitate [1]. Although no TEM was performed on sample $C$, we suppose the pinning nanostructure to be very similar to that for sample B1 due the similarity between the heat treatment schedules and the $\mathrm{J}_{\mathrm{ct}}$ properties of both wires.

\section{B. $J_{c}$ Transport and Magnetization Measurements}

Samples A, B and C were studied by means of transport and magnetization measurements. The magnetization measurements were performed at $4.2 \mathrm{~K}$ and $6 \mathrm{~K}$ for measurements. The magnetic field was always applied normal to the wire axis.

The transport critical current $\left(\mathrm{I}_{\mathrm{c}}\right)$ was measured at $4.2 \mathrm{~K}$ with voltage taps spaced 22 or $33 \mathrm{~cm}$ apart, using a resistivity criteria of $10^{-14} \Omega-\mathrm{m}$. $\mathrm{J}_{\mathrm{ct}}$ was determined by dividing the $\mathrm{I}_{\mathrm{c}}$ by the superconducting filament cross-sectional area.

Magnetic moment measurements were made using a SQUID magnetometer. The SQUID instrument has a $5 \mathrm{~mm}$ usable sample space diameter $\left(d_{b}\right)$ normal to the applied field. In the configuration of applied magnetic field perpendicular to the wire axis, the current is induced to flow along the
TABLE I

APC (SAMPLE A) AND CONVENTIONAL (SAMPLES B 1, B2 AND C) Nb-Ti WIRE CHARACTERISTICS

\begin{tabular}{|c|c|c|c|c|}
\hline \multirow[b]{2}{*}{ Sample } & \multirow[b]{2}{*}{$t_{p}(n m)$} & \multirow[b]{2}{*}{$d_{f}(\mu m)$} & \multicolumn{2}{|c|}{$A_{w}$} \\
\hline & & & SQUID & Transport \\
\hline \multirow{5}{*}{ A } & \multirow{5}{*}{$2-19^{\mathrm{a}}$} & \multirow{5}{*}{66} & $-1=$ & 5000 \\
\hline & & & 2380 & $\ldots$ \\
\hline & & & 610 & $\ldots$ \\
\hline & & & 151 & $\ldots$ \\
\hline & & & 30 & \\
\hline \multirow{5}{*}{$\mathrm{Bl}$} & \multirow{6}{*}{$2^{a}$} & \multirow{6}{*}{63} & $\ldots$ & 5240 \\
\hline & & & 1140 & $\ldots$ \\
\hline & & & 760 & $\ldots$ \\
\hline & & & 380 & $\ldots$ \\
\hline & & & 130 & $\ldots$ \\
\hline \multirow{3}{*}{$\mathrm{B} 2$} & & & 32 & $\ldots$ \\
\hline & \multirow[t]{2}{*}{$9^{a}$} & \multirow[t]{2}{*}{161} & $\ldots$ & 2050 \\
\hline & & & 298 & $\ldots$ \\
\hline \multirow[t]{2}{*}{$\mathrm{C}$} & \multirow[t]{2}{*}{$\ldots$} & \multirow[t]{2}{*}{22.3} & $\ldots$ & 14800 \\
\hline & & & 1970 & $\ldots$ \\
\hline
\end{tabular}

$t_{p}$ is the pinning center thickness, $d_{l}$ is the superconducting filament diameter and $A_{W}=L / d_{f}$ is the filament aspect ratio

a estimated from TEM analysis performed on the same APC [7] and conventional [1] wires at smaller diameters

length of the filaments and to return by crossing the filaments at each end of the wire. Two sample configurations were used. For the case of $\mathrm{L}<\mathrm{d}_{\mathrm{b}}$, many straight wire pieces were used in order to obtain a large signal. For $\mathrm{L}>\mathrm{d}_{\mathrm{b}}$, the wire was wound into an open circuit coil with an outer diameter of just less than $\mathrm{d}_{\mathrm{b}}$. SQUID magnetization data were taken continuously in the field range from $0-5 \mathrm{~T}$.

\section{Calculation Of J! From Magnetization Hysteresis IN ANISOTROPIC SAMPLES}

Recently, Sumption [6] derived expressions for $\Delta \mathrm{M}$, the width of the magnetization hysteresis for cylindrical-shaped samples having anisotropic critical current densities $\mathbf{J}_{\|}, J_{\perp}$ in a field applied normal to the cylinder axis and aspect ratio $A_{w}$ $\left(\mathrm{L} / \mathrm{d}_{\mathrm{f}}\right)$. (MKS units)

$$
\begin{aligned}
\Delta M(H)= & \frac{4 J_{1 \mid} d_{f}}{3 \pi}\left(1-\frac{3 \pi d_{f}}{32 L} \frac{J_{11}}{J_{\perp}}\right) \\
& \text { when } J_{\| 1} / J_{\perp}<L / d_{f} \\
\Delta M(H)= & \frac{J_{\perp} L}{2}\left(1-\frac{4 L}{3 \pi d_{f}} \frac{J_{\perp}}{J_{\| 1}}\right) \\
& \text { when } J_{\| 1} / J_{\perp}>L / d_{f}
\end{aligned}
$$

As already discussed, $J_{\|}$is $\gg J_{\perp}$ for these wires. Equations (1) and (2) correspond to the situations of long and short wires respectively. Thus for long samples $\left(\left(3 \pi \mathrm{d}_{\mathrm{f}} / 32 \mathrm{~L}\right) \mathrm{J}_{||} / \mathrm{J}_{\perp}\right.$ $\ll 1$ so that $\left.\Delta \mathrm{M} \rightarrow 4 \mathrm{~d}_{\mathrm{f}} \mathrm{J}_{\mid} / 3 \pi\right)$ the isotropic $\mathrm{BM}$ is valid. For very short samples such that $\left(4 \mathrm{~L} / 3 \pi \mathrm{d}_{\mathrm{f}}\right) \mathbf{J}_{\perp} / \mathbf{J}_{\|} \ll 1, \mathrm{M}$ is de- 
termined by the perpendicular current density $J_{\perp}$ and the sample length $L$.

\section{RESULTS AND DISCUSSION}

Figs. $1 \mathrm{a}$ and $1 \mathrm{~b}$ show $\Delta \mathrm{M}(6 \mathrm{~K})$ vs. $\mathrm{H}$ curves for samples $\mathrm{A}$ and $\mathrm{B} 1$. Both wires have anisotropic critical current densities, indicated by the increase of $\Delta \mathrm{M}$ as $\mathrm{L}$ increases. $\Delta \mathrm{M}$ saturates at $L \geq 40 \mathrm{~mm}$ for $A$ and for $L \geq 48 \mathrm{~mm}$ for $B 1$.

In order to compare the saturation magnetization values to the transport measurements, which could only be measured at $4.2 \mathrm{~K}$, magnetization measurements were made on the same lengths of $\mathrm{A}$ at both temperatures. The saturation length was the same at both temperatures. Fig. 2 shows the bulk pinning force density curves $\mathrm{F}_{\mathrm{p}}(\mathrm{H}, 4.2 \mathrm{~K})$ calculated from $\mathrm{J}_{\mathrm{ct}}$ and from $J_{\mid l}$ (SQUID). As shown in Fig. 2, there is no significant difference between the pinning force curves constructed from $\mathrm{J}_{\mathrm{ct}}$ and $J_{\|}$calculated from SQUID measurements of $M_{s} . \quad F_{p}$ peaks at $2.2-2.4 \mathrm{~T}$ for both measurements and the magnitudes of $F_{p}$ differ by only $4 \%$ at $2.3 \mathrm{~T}$. Such small differences are within the experimental uncertainty.

By contrast, there is no such agreement between the same measurements made on the conventional wire. There is always a significant difference between $\mathrm{J}_{\|}$and $\mathrm{J}_{\mathrm{ct}}$ and, at $5 \mathrm{~T}$, the magnitudes of $\mathrm{J}_{\mathrm{ct}}$ and $\mathrm{J}_{\|}$differ by $20 \%$. Indeed, the same kind of magnetization-length-dependence in another optimized conventional $\mathrm{Nb}$ - $\mathrm{Ti}$ wire (sample $\mathrm{C}$ ) showed the same effect and the difference in magnitude between $\mathrm{J}_{\mathrm{ct}}$ and $\mathrm{J}_{\mid \uparrow}$ at 5 $T$ reached $100 \%$ in this case [9].

Being concerned that the discontinuity of the $\alpha$-Ti ribbons in conventional $\mathrm{Nb}-\mathrm{Ti}$ might be playing a role in the SQUID measurements, we measured wire $B$ at a larger diameter (sample B2) where the $\alpha$-Ti pins are less ribbon-like, thicker and shorter than in the optimized wire (B1). Fig. 3 shows that $J_{c t}$ and $J_{\|}$are still noticeably different. Furthermore the differences in magnitude and field dependence are higher for sample B2 than for sample B1. The $F_{p}\left(J_{\mid}\right)$curves start to differ from $\mathrm{F}_{\mathrm{p}}\left(\mathrm{J}_{\mathrm{ct}}\right)$ at $2.5 \mathrm{~T}$ and $1 \mathrm{~T}$ for samples B1 (Fig. 1b) and B2 (Fig. 3) respectively. For sample B2, the peaks of $F_{p}\left(J_{c t}\right)$ and $F_{p}\left(J_{\mid l}\right)$ are at $3.5 \mathrm{~T}$ and $2.8 \mathrm{~T}$ each and the magnitudes differ by more than $30 \%$ at $5 \mathrm{~T}$.

$\Delta \mathrm{M}(\mathrm{L})$ vs. $\mathrm{H}$ measurements on $\mathrm{B} 2$ showed that the magnetization saturates for $75<\mathrm{A}_{\mathrm{w}}<224$ and at $381<\mathrm{A}_{\mathrm{w}}<762$ for B1. These differences point to the fact that the lengths of the ribbons in conventional $\mathrm{Nb}-\mathrm{Ti}$ wires play an important role on the saturation of $\Delta \mathrm{M}(\mathrm{L})$ vs. $\mathrm{H}$ because they are directly related to the wire lengths $L_{i} \rightarrow L$ and $d_{i} \rightarrow d_{f}$. We suggest that the differences between $\mathrm{J}_{\mathrm{ct}}$ and $\mathrm{J}_{\|}$are related to the low electric field attainable in SQUID measurements which allows the induced-magnetization-currents to redistribute around the discontinuous ribbons in conventional $\mathrm{Nb}-\mathrm{Ti}$ wires. Consequently, the larger differences in magnitude between $F_{p}\left(J_{c t}\right)$ and $F_{p}\left(J_{1}\right)$ observed for sample B2 compared to sample B 1 can be explained using equation (1). Sample B 1 has a larger ribbon aspect ratio $L_{i} / d_{i}\left(\right.$ strictly $\left.d_{i}=t_{p}\right)$ than sam-
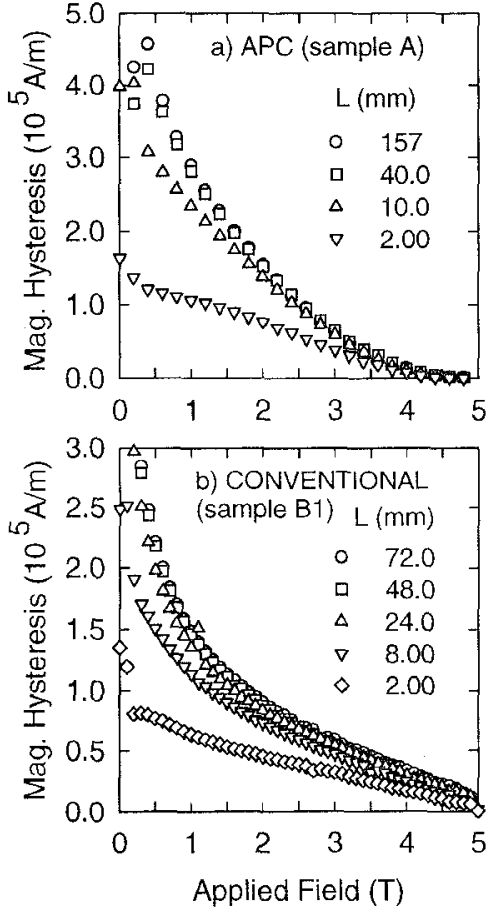

Fig. 1. $\Delta \mathrm{M}(\mathrm{H})$ width increase and saturation with an increasing wire length $L$ for a) Sample A and b) Sample B1, measured in a SQUID magnetometer at $6 \mathrm{~K}$.

ple B2. From equation (1), the difference between $J_{c t}$ and $J_{\|}$ should be smaller for sample B1 than for sample B2 because

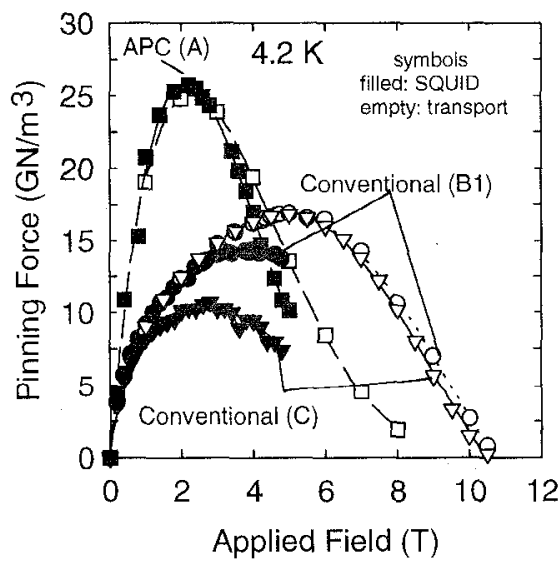

Fig. 2. Pinning force density curves as a function of the applied magnetic field at $4.2 \mathrm{~K}$ showing the differences in critical current density obtained by different measurement techniques for APC and conventional $\mathrm{Nb}-\mathrm{Ti}$ wires (B1 and C). 


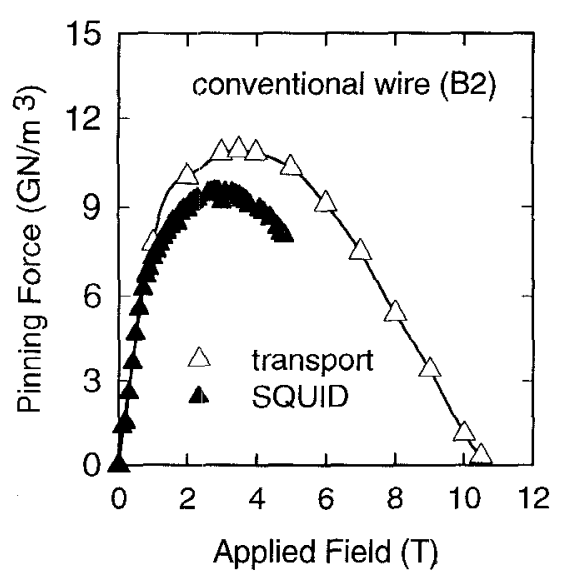

Fig. 3. Pinning force density curves as a function of the applied magnetic field showing the differences in critical current density obtained from $\operatorname{SQUID}\left(\Delta \mathrm{M}_{\mathrm{s}}\right)$ and transport measurements of $\mathrm{B} 2$.

$\mathrm{d}_{\mathrm{f}} / \mathrm{L}(\mathrm{B} 1)<\mathrm{d}_{\mathrm{f}} / \mathrm{L}(\mathrm{B} 2)$. The term $\left(3 \pi \mathrm{d}_{\mathrm{f}} / 32 \mathrm{~L}\right) \mathrm{J}_{||} / \mathrm{J}_{\perp}$ will be close to 1 for sample $B 2$ and $\Delta \mathrm{M}_{\mathrm{s}}$ should then be only a small fraction of $J_{c t}=J_{\|}$. In this analysis we considered $J_{\|} / J_{\perp}$ constant for different ribbon dimensions. This is reasonable because we have already shown in [5] that the magnitudes of $J_{||} / J_{\perp}$ are comparable for thin and thick APC ribbons.

\section{COMPARISON OF THE MAGNETIZATION CURRENTS ANISOT- ROPY BETWEEN APC AND CONVENTIONAL Nb-Ti WIRES}

As was already mentioned, it was not possible to extract $J_{||} / J_{\perp}$ for conventional wires from the $\Delta M(L)$ vs. $H$ measurements. However, we can make a comparison between the anisotropy of conventional and APC wires by calculating the ratio of the saturated magnetization hysteresis $\Delta M_{s}$ to the magnetization of the shortest measured wire lengths, because $\Delta \mathrm{M}_{\mathrm{s}}$ is determined by $\mathrm{d}_{\mathrm{f}}$ and $\mathrm{J}_{\|}\left(=\mathrm{J}_{\mathrm{ct}}\right)$. Equation (2) shows that $\Delta \mathrm{M}$ is dominated by $\mathrm{J}_{\perp}$ and $\mathrm{L}$ for short wire lengths. Samples $A$ and $B 1$ have similar $d_{f}$ values and were measured at $\mathrm{L}=2 \mathrm{~mm}$. Therefore $\Delta \mathrm{M}_{\mathrm{s}} / \Delta \mathrm{M}(2 \mathrm{~mm}) \propto\left(\mathrm{J}_{||} / \mathrm{J}_{\perp}\right)\left(\mathrm{d}_{\mathrm{f}} / \mathrm{L}\right)$ and as $\mathrm{d}_{\mathrm{f}} / \mathrm{L}$ is the same for both wires, the described magnetization hysteresis ratio permits a good comparison of the anisotropy between conventional and $\mathrm{APC} \mathrm{Nb}-\mathrm{Ti}$ wires. For the conventional wire, we calculated $\Delta \mathrm{M}_{\mathrm{s}}=4 \mathrm{~J}_{\mathrm{ct}} \mathrm{d}_{\mathrm{f}} / 3 \pi$. In Fig. 4 the curves of $\Delta \mathrm{M}_{\mathrm{s}} / \Delta \mathrm{M}(2 \mathrm{~mm})$ vs. $\mathrm{H}$ for $\mathrm{APC}$ and conventional wires at $4.2 \mathrm{~K}$ are shown. A markedly higher anisotropy and stronger field dependence at $<3 \mathrm{~T}$ is apparent for the APC wire, while the anisotropy for the conventional wire is almost constant up to $5 \mathrm{~T}$. This difference in behavior could be explained by the wider distribution of pin thickness in the APC

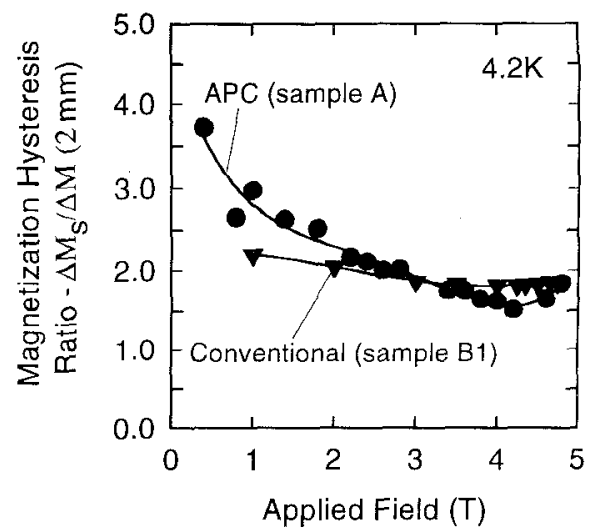

Fig. 4. Ratio of magnetization hysteresis $\Delta \mathrm{M}_{\mathrm{s}} / \triangle \mathrm{M}(\mathrm{L}=2 \mathrm{~mm})$ vs. $\mathrm{H}$ for APC (sample A) and conventional (sample B1) wires at $4.2 \mathrm{~K}$.

wire that should influence the field dependence of $J_{\perp}$. For fields higher than $3 \mathrm{~T}$, the conventional wire is slightly more anisotropic than the $\mathrm{APC}$ wire.

\section{SUMMARY}

There are subtle but important differences between the properties of $\mathrm{APC}$ and conventional $\mathrm{Nb}-\mathrm{Ti}$ wires. A new insight is that the distinction between continuous pins (APC) and discontinuous pins (conventional) is important for understanding magnetization measurements. It seems likely that local current loops circulating between pins are possible in conventional wires.

\section{REFERENCES}

[1] C. Meingast, P.J. Lee and D.C. Larbalestier, J. Appl. Phys., vol. 66 (12), 5962-5970, 1989; C. Meingast and D.C. Larbalestier; J. Appl. Phys., vol. 66 (12), 1989 , pp. $5971-5983$.

[2] L.R. Motowidlo, B.A. Zeitlin, M.S. Walker and P. Haldar, Appl. Phys. Lett. vol. 61, 1992, pp. 991-993.

[3] K. Matsumoto, H. Takewasi, Y. Tanaka, O. Miura, K. Yamafuji, K. Funaki, M. Iwakuna and T. Matsushita, Appl. Phys. Lett., vol. 64, 1994, pp. 115-117.

[4] R.W. Heussner, P.D. Jablonski, P.J. Lee and D.C Larbalestier, IEEE Trans. Appl. Supercond., vol. 5, 1995, pp. 1705-1708.

[5] C. Bormio Nunes, R.W. Heussner and D.C. Larbalestier, I. Appl. Phys. vol. 80 (3), 1996, pp. 1647-1651.

[6] M. Sumption, Appl. Superconductivity, vol. 2 (1), 1994, pp. 41-46.

[7] C.P. Bean, Rev. Mod. Phys. vol. 36, 1964, pp. 31.

[8] R.W. Heussner, C. Bormio Nunes, P.J. Lee, P.D. Jablonski and D.C. Larbalestier, J. Appl. Phys. vol, 80 (3), 1996, pp. 1640-1646.

[9] C.B. Nunes, unpublished data. 